\title{
Monadic Printing Revisited
}

\author{
Konrad Grzanek \\ IT Institute, University of Social Sciences \\ 9 Sienkiewicza St., 90-113 Łódź, Poland \\ kgrzanek@spoleczna.pl
}

\begin{abstract}
Expressive and clear implementation of monadic printing requires an amount of work to define and design proper abstractions to rely upon when performing the actual programming works. Our previous realization of tree printing library left us with a sense of lack with respect to these considerations. This is why we decided to re-design and re-implement the library with core algorithms based upon new, effective and expressive text printing and concatenation routines. This paper presents the results of our work.
\end{abstract}

Keywords: Functional programming, monads, Haskell, polymorphism

\section{Introduction}

Textual presentation of data structures is invariably one of the most effective ways to visualize them, especially when it comes to presentation of large data structures. The ability to display textual content and working on the presentation results with automated text-processing tools sometimes makes this way of visualizing much more appealing to the end-user than displaying using GUI views. The data structure that is especially susceptible to this approach is tree, or - even more generally - DAG (Directed Acyclic Graph).

Our previous work on this subject aimed towards creating a library for visualizing trees and DAGs. Our few years old paper [5] presented a library for Haskell [1, 2], the purely functional and statically typed programming language. The library described there possessed the following properties:

- The ability to generate representations of arbitrary DAGs.

- Writing to any monad including IO. This also means it was capable of writing to normal Haskel Strings (lists of Char) via Identity monad.

- Extensive use of Haskell type-system to verify correctness of the usage scenarios. 
Unfortunately, the design and implementation of this library was not perfect. It missed expressiveness and the clarity of algorithm formulation. These issues led to extensive re-design of the library. The updated architecture of the library consists of:

- Printing abstraction,

- String/Text concatenation routines,

- Re-designed tree printing implemented on top of the two previous ones.

This paper is an attempt to present all the details of the refreshed library.

\section{Printing Abstraction and Its Implementations}

Generic printing mechanisms are defined in Kask.Print module [6]. All its contents are defined in the presence of the following import clauses:

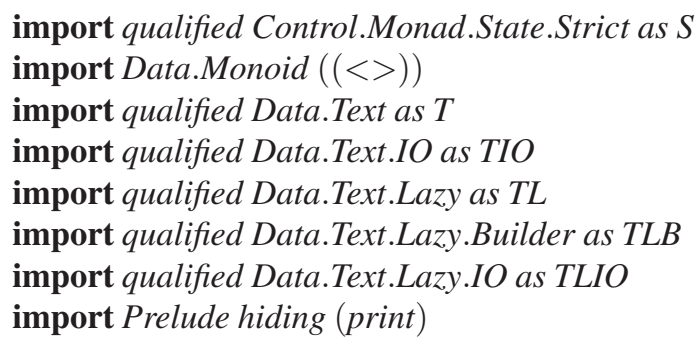

The most essential abstraction is a type-class called Printable. It is parameterized by two type-arguments out of which the first one, $m$, is a monad [4].

We have two procedures defined here, namely print and print $L n$. They both return a unit-type in the monad $m$. The printLn works exactly like print, but it adds a newline character to the end of the printed entity of type $p$ :

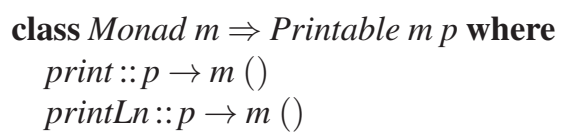

\subsection{IO Monad}

The Printable type-class is implemented within the IO monad for a collection of textual data-types, like String, ShowS, and Text, either lazily and eagerly evaluated. See the listing below:

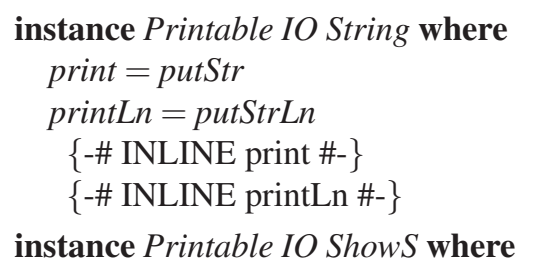




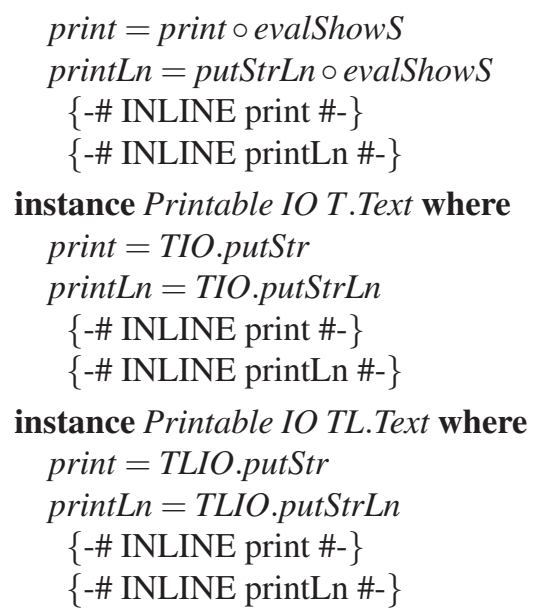

We also provide an IO-monadic implementation for an effective textual builder defined in Data.Text.Lazy.Builder, like:

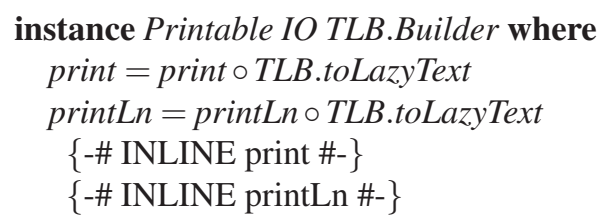

\subsection{Text in the State Monad}

Another interesting monad to mention here is the state monad, as defined in Control.Monad.State.Stric. We define a special type TextBuilder to wrap the textual state management within an useful text-coercible abstraction:

$$
\begin{aligned}
& \text { type TextBuilder }=\text { S.State T.Text } \\
& \text { toText }:: \text { TextBuilder }() \rightarrow \text { T.Text } \\
& \text { toText } t b=\text { snd }(\text { S.runState } t b ") \\
& \quad\{-\# \text { INLINE toText } \# \text { - }\}
\end{aligned}
$$

The TextBuilder monad has the following Printable implementations for String and ShowS:

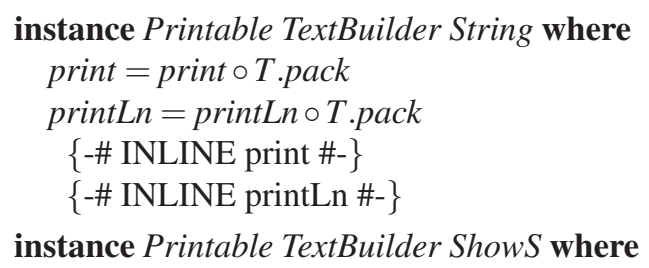




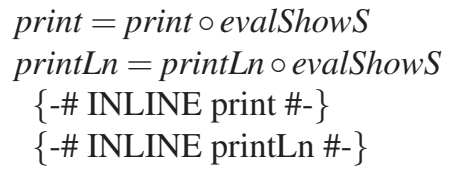

as well as for eagerly, and lazily evaluated Text:

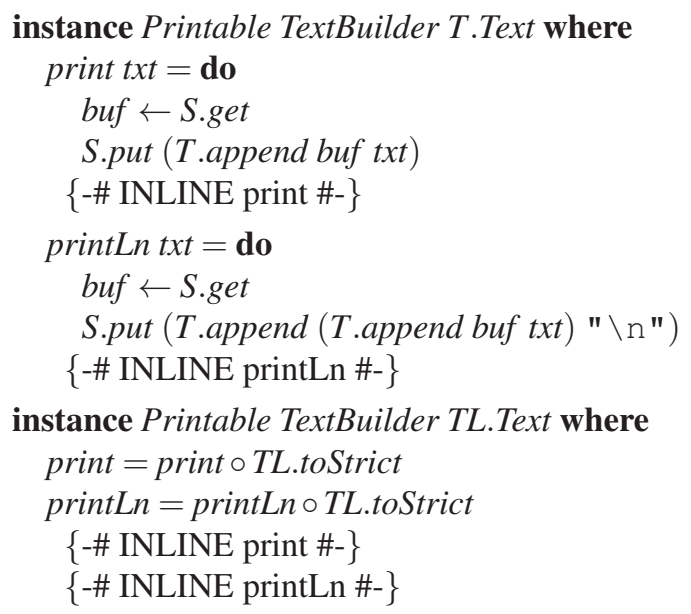

We also provide implementation for Data.Text.Lazy.Builder like in the case of IO monad:

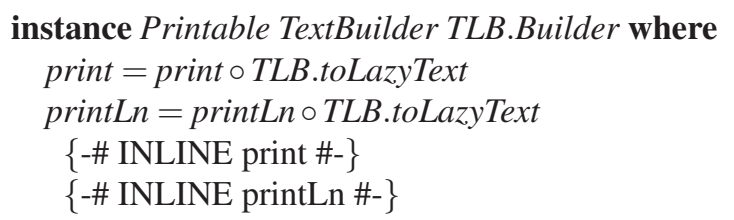

\subsection{Lazy Text Builder in the State Monad}

Eagerly evaluated state monad may be also used as a basis for a lazily evaluated string builder, as defined below, together with two state evaluators:

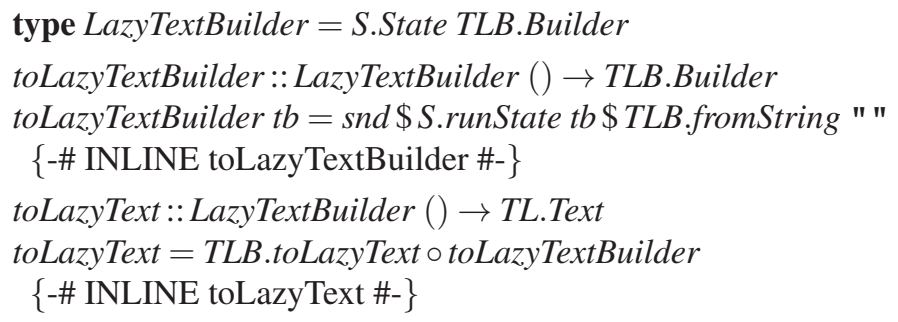

Like in the case of the previous monadic implementations, firstly we define the implementations for String and ShowS: 


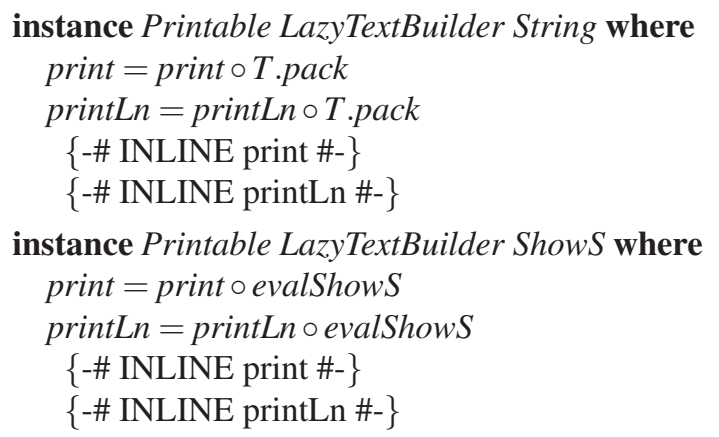

as well as for strictly and lazily evaluated Text instances:

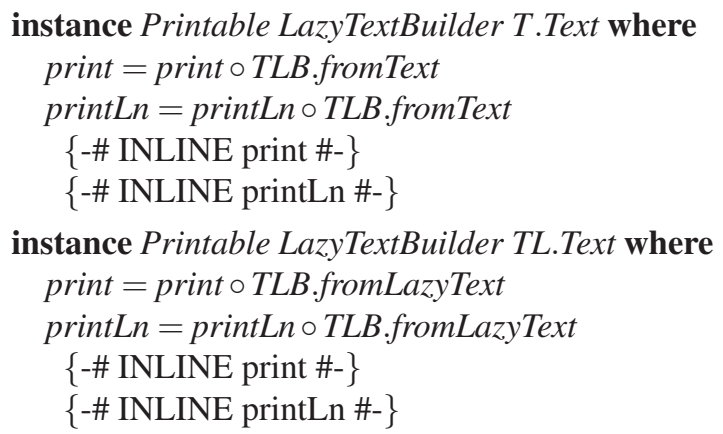

To make this realization conceptually coherent with the previous ones, we also provide an implementation for TLB.Builder (as it was presented in the previous sub-sections):

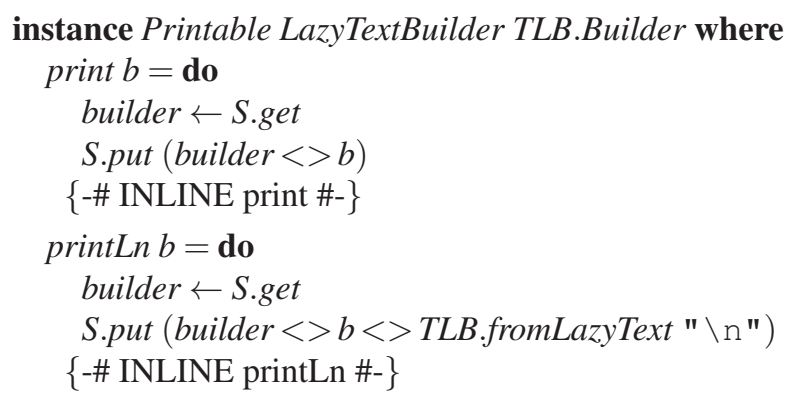

\subsection{ShowS in the State Monad}

For ShowS type we define a separate State Monad instance, together with the following evaluators:

type StringBuilder $=$ S.State ShowS
evalShowS $::$ Show $S \rightarrow$ String 


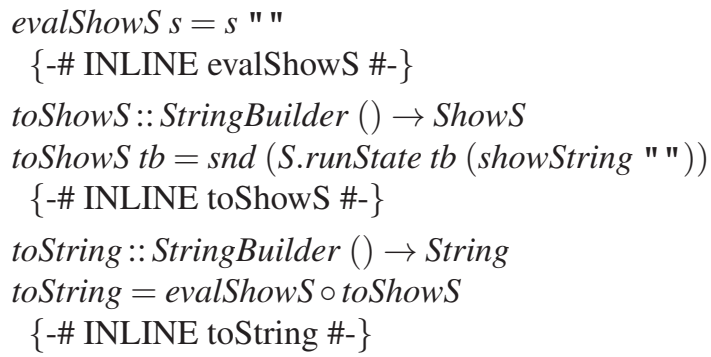

The String and ShowS instances of the Printable type-class raise up in a natural way:

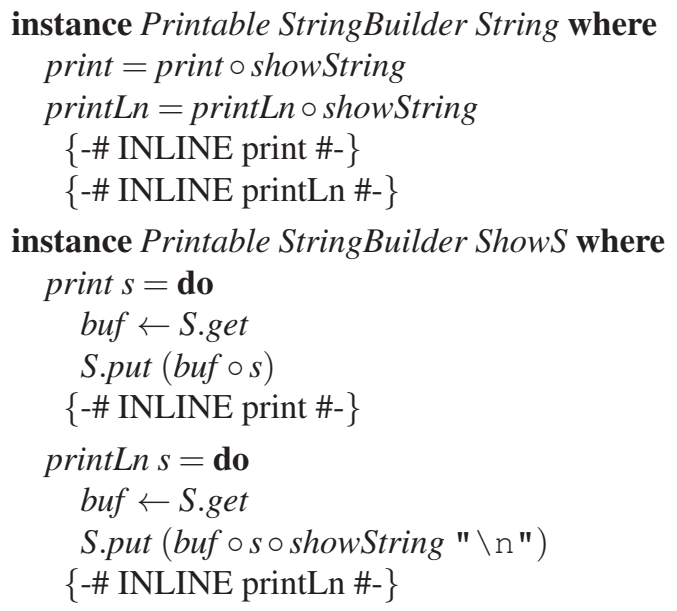

along with Text instances, like in the following listing:

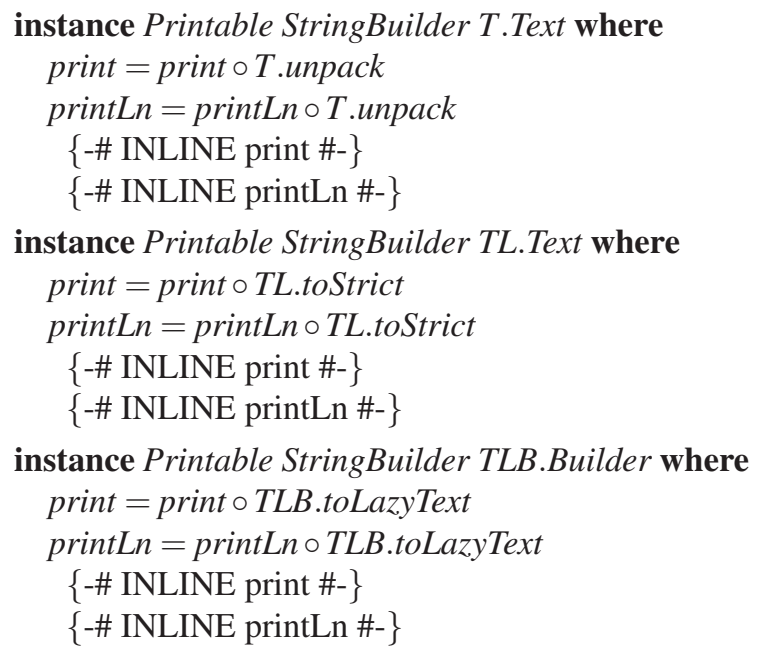




\section{Compatible Abstraction for Concatenation}

Early in the design phase it became apparent that we might use the Printable for string concatenation. After all the concatenation may be viewed here as printing into the concatenating (string/text builder) object. To make things clear we provide the following StrCat type-class, that is another useful abstraction in our library:

\section{class StrCat $c$ where}

$$
\text { strCat:: }(\text { Foldable } t) \Rightarrow t c \rightarrow c
$$

Concatenation is being treated as a fold (e.g. see [3]) operation, that's why we define the strCat mechanism as taking place inside a Foldable.

Functional merging of StrCat and Printable takes place via the following strCatWith procedure:

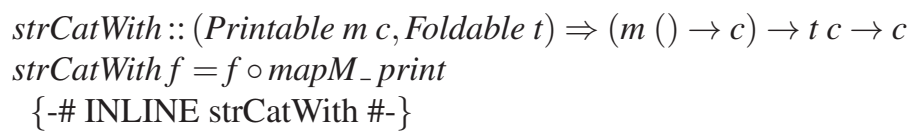

This immediately allows us to provide StrCat implementations for String and ShowS:

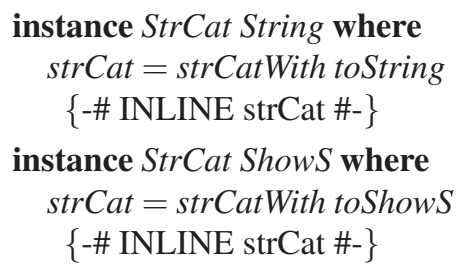

The same approach applies to Text and TLB.Builder:

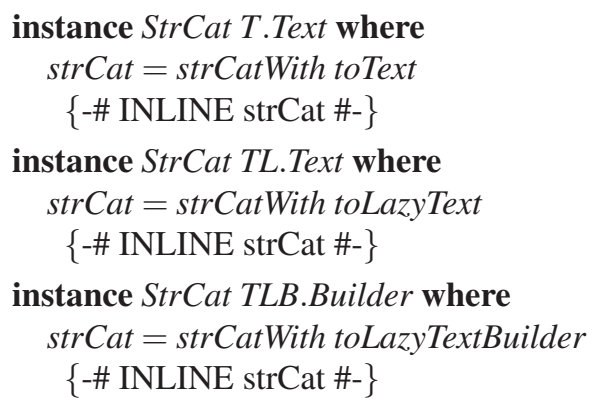

\section{Re-designed Tree Printing}

All abstractions and their implementations described so far allow us to provide an updated realization of tree printing, previously defined and presented in [5]. The new 
realization can be viewed as a whole in Kask.Data.Tree.Print module [7]. In the presence of the following import clauses:

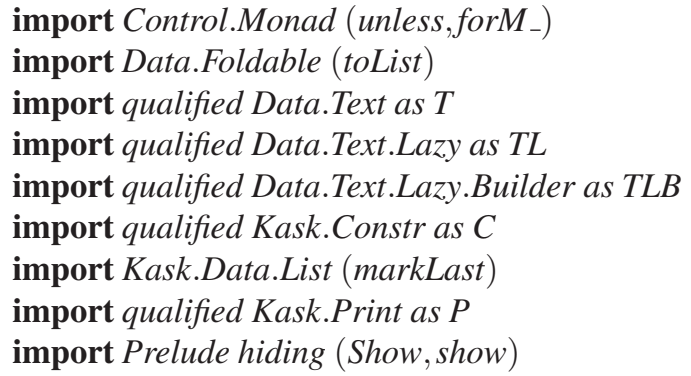

we have the basic type definitions like below:

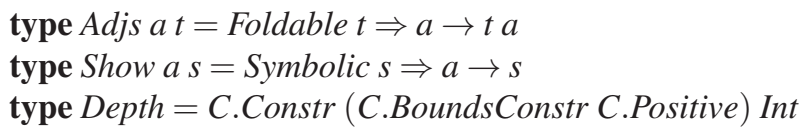

One additional visible change with respect to mechanisms defined in [5] relates to Depth - a new data type that describes the maximum depth of tree-printing. Currently it is a positive integer, with the contract enforced by using Constr and BoundsConstr, an effective compile-time contract definition routines, also provided by the kask repository.

\subsection{Tree Printing API}

Essentially it consists of a single procedure printTree with the following signature and implementation:

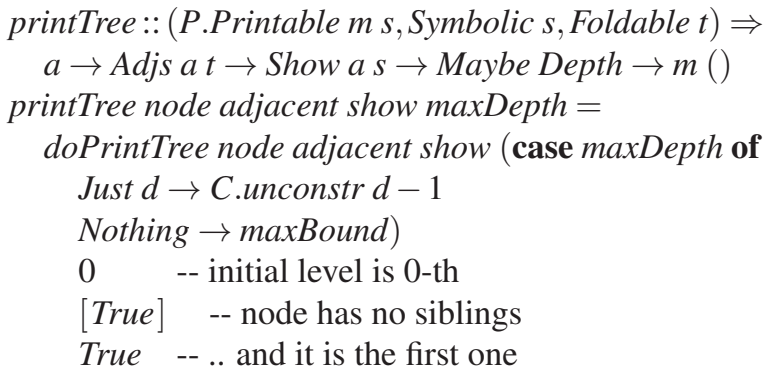

\subsection{Simplified and More Expressive Tree Printing Implementation}

The procedure takes the following form:

$$
\begin{aligned}
& \text { doPrintTree }::(\text { P.Printable } m \text { s, Symbolic } s, \text { Foldable } t) \Rightarrow \\
& \quad a \rightarrow \text { Adjs a } t \rightarrow \text { Show a } s \rightarrow \text { Int } \rightarrow \text { Int } \rightarrow[\text { Bool }] \rightarrow \text { Bool } \rightarrow m()
\end{aligned}
$$




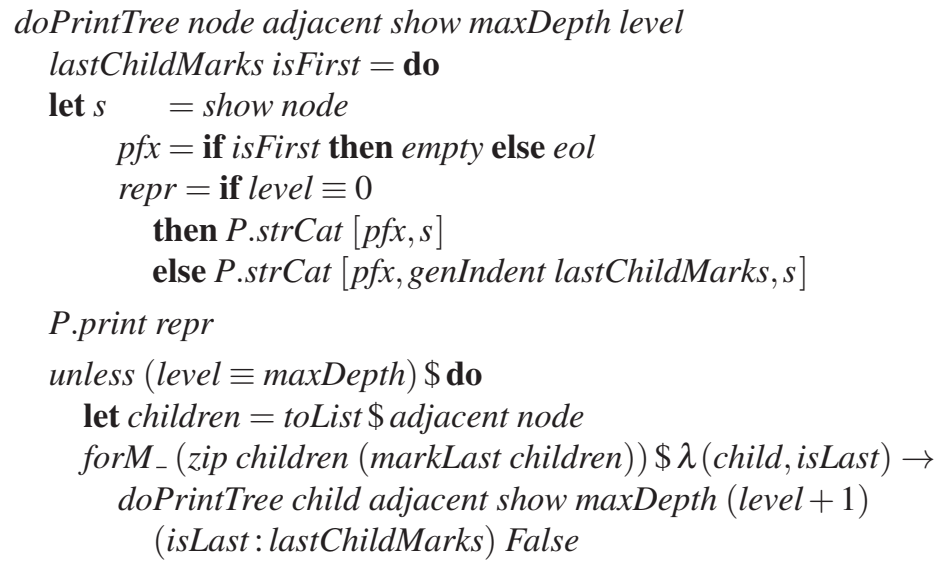

All the printing, concatenation and string-building abstractions allowed us to achieve two goals:

1. Make the implementation clear and obvious.

2. Make the API expressive.

The clarification seems apparent here, and the expressiveness enhancement takes place thanks to powerful compile time abstractions provided in the signature: Printable, Foldable, Adjs, Show.

\subsection{Further Implementation Details}

String concatenation abstraction is also used to implement properly the indentation used to layout the printed tree:

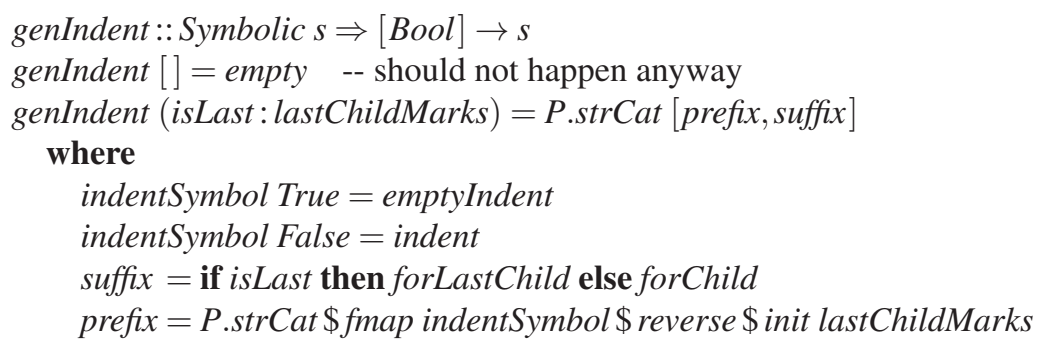

Additionally we use a Symbolic type class that holds the information about all textual elements forming the tree printing layout. The abstraction is defined as:

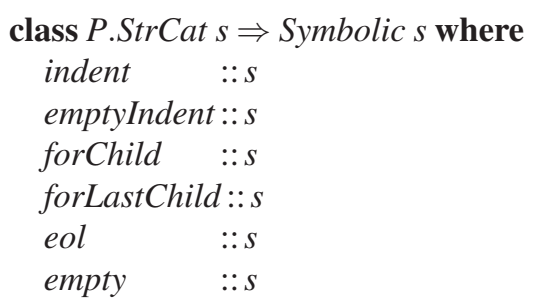


with the following realization for String and ShowS:

instance Symbolic String where

indent $=" \quad "$
emptyIndent $="$
forChild $=" \quad "$
forLastChild $=" \quad "$
eol $=" \backslash \mathrm{n} "$
empty $=" "$

instance Symbolic Shows where

$$
\begin{aligned}
& \text { indent } \quad=\text { showString (indent }:: \text { String) } \\
& \text { emptyIndent }=\text { showString }(\text { emptyIndent }:: \text { String }) \\
& \text { forChild = showString (forChild :: String) } \\
& \text { forLastChild }=\text { showString (forLastChild :: String) } \\
& \text { eol = showString (eol ::String) } \\
& \text { empty = showString (empty ::String) }
\end{aligned}
$$

Finally, we also provide an implementation for Text:

$$
\begin{aligned}
& \text { instance Symbolic T.Text where }
\end{aligned}
$$

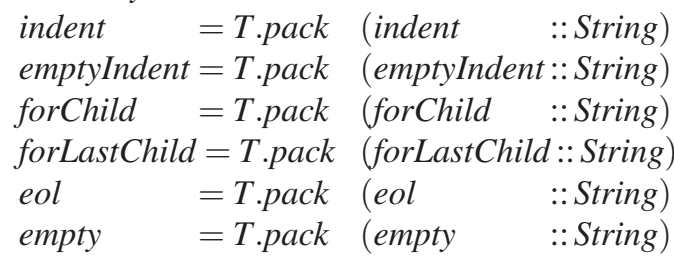

instance Symbolic TL.Text where

$$
\begin{aligned}
& \text { indent }=\text { TL.pack (indent ::String) } \\
& \text { emptyIndent }=\text { TL.pack (emptyIndent }:: \text { String }) \\
& \text { forChild = TL.pack (forChild :: String) } \\
& \text { forLastChild }=\text { TL.pack (forLastChild:: String) } \\
& \text { eol } \quad=\text { TL.pack (eol ::String) } \\
& \text { empty =TL.pack (empty ::String) }
\end{aligned}
$$

and for TLB.Builder:

$$
\begin{aligned}
& \text { instance Symbolic TLB.Builder where } \\
& \text { indent }=\text { TLB. fromText (indent ::T.Text) } \\
& \text { emptyIndent }=\text { TLB. fromText }(\text { emptyIndent }:: \text { T.Text }) \\
& \text { forChild = TLB.fromText (forChild } \quad:: T \text {.Text) } \\
& \text { forLastChild }=T L B \text {. fromText }(\text { forLastChild }:: T \text { Text }) \\
& \text { eol } \quad=\text { TLB.fromText (eol ::T.Text) } \\
& \text { empty } \quad=\text { TLB.fromText (empty } \quad:: T . T e x t)
\end{aligned}
$$


Grzanek K.

\section{References}

1. Peyton Jones S., 1987, The Implementation of Functional Programming Languages, Prentice-Hall International Series in Computer Science. Prentice Hall International (UK) Ltd

2. Lipovaca M., 2011, Learn You a Haskell for Great Good!: A Beginners Guide, No Starch Press; 1st edition (April 21, 2011)

3. Bird R., Wadler R., 1988, Introduction to Functional Programming. Series in Computer Science (Editor: C.A.R. Hoare), Prentice Hall International (UK) Ltd

4. Awodey S., 2010, Category Theory, Second Edition, Oxford University Press

5. Grzanek K., 2014, Monadic Tree Print, JACSM 2014, Vol. 6, No. 2, pp. 147-157

6. GitHub, 2016, Kask.Print module: Kask repository, https://github.com/kongra/kask/blob/master/src/Kask/Print.hs

7. GitHub, 2016, Kask.Data.Tree.Print module: https://github.com/kongra/kask/blob/master/src/Kask/Data/Tree/Print.hs 